\title{
LI. Note on the double refraction of electric waves
}

\section{George Pierce Ph.D.}

To cite this article: George Pierce Ph.D. (1901) LI. Note on the double refraction of electric waves, Philosophical Magazine Series 6, 1:5, 548-551, DOI: 10.1080/14786440109462643

To link to this article: http://dx.doi.org/10.1080/14786440109462643

册 Published online: 08 Jun 2010.

Submit your article to this journal 준

Џ Article views: 2

Q View related articles $₫$ 
directly with the bands obtained in vacuo or in air for the same path. The results of experiments now in progress demonstrate the greater simplicity and accuracy of the method as compared with others.-D. B. BRACE.]

Physical Laboratory,

University of Nebraska, Lincoln.

\section{Note on the Double Refraction of Electric Waves.} By George Pierce, Ph.D.*

IN

$N$ the February number of the Philosophical Magazine I have described an experiment on the measurement of the refractive indices for electric waves of a number of doublyrefractive media: namely, various woods. These media were all also doubly-absorptive.

In the present brief note I wish to examine the question as to whether, on Maxwell's theory, the double absorption by these media is sufficient to account for the double refraction; and, indeed, whether both of these properties can be ascribed merely to differences of conductivity of the woods along and across the grain. The treatment that follows, except the application, is taken largely from Boltzmann's Vorlesungen über Mawuells Theorie, \$96 (Leipzig, 1891).

Let $f, g, h$ be the components of electric polarization; then in a conducting medinm, of conductivity $\mathrm{C}$, the equations of the field are (Boltzmann eq. 81)

$$
\mu k \frac{d^{2} f}{d t^{2}}+4 \pi \mu \mathrm{C} \frac{d f}{d t}=\frac{d^{2} f^{2}}{d x^{2}}+\frac{d^{2} f}{d y^{2}}+\frac{d^{2} f}{d z^{2}}, \quad . \quad .
$$

and similar equations for $g$ and $h$.

Now let us assume a plane wave propagated in the direction of the Z-axis, and let the electric displacement be in the direction of the X-axis ; then $g=h=0$, and $f$ is a function of $t$ and $z$ alone; whence equation (1) becomes

$$
\mu k \frac{d^{2} f}{d t^{2}}+4 \pi \mu \mathrm{C} \frac{d f}{d t}=\frac{d^{2} f}{d z^{2}} . \quad . \quad . \quad .
$$

A particular solution of (2) is

$$
f=\mathrm{B} e^{\frac{2 \pi}{\mathrm{T}} t i+(\xi+\gamma i) z}
$$

where B, T, $\xi$. and $\eta$ are constants, to determine which we

$$
\text { * Communicated by the Author. }
$$



must substitate this value of $f$ in $(2) . \quad W$ riting $V_{0}=\frac{1}{\sqrt{k \mu}}$, we
obtain

$$
\begin{aligned}
& \xi^{2}=-\frac{2 \pi^{2}}{\mathrm{~T}^{2} \mathrm{~V}_{0}^{2}}+\sqrt{\frac{4 \pi^{4}}{\mathrm{~T}^{4} \mathrm{~V}_{0}{ }^{4}}+\frac{16 \pi^{4} \mathrm{C}^{2} \mu^{2}}{\mathrm{~T}^{2}}}, \\
& \eta^{2}=+\frac{2 \pi^{2}}{\mathrm{~T}^{2} \mathrm{~V}_{0}{ }^{2}}+\sqrt{\frac{4 \pi^{4}}{\mathrm{~T}^{4} V_{0}{ }^{4}}+\frac{16 \pi^{4} \mathrm{I}^{2} \mu^{2}}{\mathrm{~T}^{2}}} .
\end{aligned}
$$

The solution (3) is imaginary, but two such solutions properly combined give, as a real solution of (2),

$$
f=\mathrm{A} e^{\xi z} \cdot \cos \left(\frac{2 \pi t}{\mathrm{~T}}+\eta z\right) .
$$

The factor $e^{\xi z}$ gives the amplitude of the wave; and $\frac{2 \pi}{\eta T}=\mathrm{V}$, the velocity of propagation.

Whence

$$
\mathrm{V}=1 / \sqrt{\frac{1}{2 \mathrm{~V}_{0}^{2}}+\sqrt{\frac{1}{4 V_{0}{ }^{4}}+\mathrm{C}^{2} \mu^{2} \mathrm{~T}^{2}}} ;
$$

When $C^{2}$ is negligible in comparison with $C(i . e$. when the conductivity is small), this expression expanded gives

$$
\mathrm{V}=\mathrm{V}_{0}-\frac{\mathrm{V}_{0}{ }^{5} \mathrm{C}^{2} \mu^{2} \mathrm{~T}^{2}}{2} ; . . . . .
$$

and similarly,

$$
\xi=-2 \pi \mathrm{C}^{\prime} \mu \mathrm{V}_{0} . \quad \text {. . . . . . }
$$

The units here used are the electromagnetic system; hence $\mathrm{V}_{0}$, from its definition $\left[-\frac{1}{\sqrt{k \mu}}\right]$, is the velocity with which the wave would move in the given medium if its conductivity were zero.

Now let $D_{0}=$ the maximum amplitude of a wave on entering a sheet of the dielectric of thickness $z_{0}$; $\mathrm{D}=$ maximum amplitude of wave on emerging.

Then $\mathrm{D}=\mathrm{D}_{0} e^{\xi z_{0}}$.

Call

$$
\frac{\mathrm{D}}{\mathrm{D}_{0}}=q
$$

Then

$$
\log q=\xi z_{0}=-2 \pi \mathrm{C} \mu \mathrm{V}_{0} z_{0} ;
$$

whence

$$
\mathrm{C}^{2} \mu^{2}=\frac{\log ^{2} q}{4 \pi^{2} \mathrm{~V}_{0}{ }^{2} z_{0}^{2}} \text {. }
$$


This value substituted in (4) gives

$$
\mathrm{V}=\mathrm{V}_{0}-\frac{\mathrm{V}_{0}{ }^{3} \mathrm{~T}^{2} \log ^{2} q}{8 \pi^{2} z_{0}{ }^{2}} \text {. . . . . . }
$$

Equation (6) is the relation between the velocity of the waves in a given weakly-conducting dielectric and their absorption by the dielectric. We are supposing here that the medium is not crystalline in the sense of having different dielectric constants along different axes, but that it is merely heterogeneous in that it has different conductivities along different axes. In a medium of this kind, $V_{0}$ is constant for a given specimen, while the absorption-coefficient $\frac{\log q}{z_{0}}$ depends upon the direction of the electric force in the specimen. In that orientation of electric force in which absorption is greatest, $\mathrm{V}$ is smallest, and therefore index of refraction is greatest.

This agrees with the results of my experiments on woods. With all the specimens tested, when the wood is so oriented that its grain is parallel to the electric displacement, the index of refraction is greater than with the grain perpendicular. In the former orientation also the absorption is greater than in the latter.

Returning now to the experimental data, let $V_{1}$ and $q_{1}$ be the values found for $V$ and $q$ with the first orientation; $V_{2}$ and $q_{2}$ the corresponding values with the second orientation. When these values are substituted in (6) we have two new equations which combined give

$$
\frac{\mathrm{V}_{1}-\mathrm{V}_{0}}{\overline{\mathrm{V}}_{2}-\mathrm{V}_{0}}=\frac{\log ^{2} q_{1}}{\log ^{2} q_{2}}
$$

Dividing numerator and denominator of the left-hand member by $v$ the velocity of the waves in air, we obtain

$$
\frac{1 / n_{1}-1 / n_{0}}{1 / n_{2}-1 / n_{0}}=\frac{\log ^{2} q_{1}}{\log ^{2} q_{2}} . \quad \text {. . . . . . }
$$

The right-hand member can be caleulated from the absorption data. Call it $\mathbf{R}^{2}$, then

$$
1 / n_{1}=\mathrm{R}^{2} / n_{2}-\left(\mathrm{R}^{2}-1\right) / n_{0} \quad . \quad . \quad \text {. }
$$

where $n_{1}=$ index of refraction in the first orientation, $n_{2}=$ index of refraction in the second orientation, and $n_{0}=\sqrt{\mu}$. 
Hence the sufficiency of the bare assumption of heterogeneous conductivity to explain the double refraction of electric waves by a medium like wood can be tested by comparing the value of $\mu$, obtained by calculation from equation (8), with the dielectric constant of the medium measured by some static method. The latter data I have not at hand.

However, without such a comparison, it is still seen that heterogeneous conductivity plays an important part in the phenomenon of double refraction of electric waves.

Universität Leipzig.

January, 1901.

LII. On the Production of a Bright-Line Spectrum by Anomalous Dispersion and its Application the "Flash-Spectrum." By R. W. Woon *.

TN a communication published in the Proceedings of the 1 Royal Academy of Sciences, Amsterdam, W. H. Julius makes the very brilliant suggestion that the "flash-spectrum," seen immediately at totality, may be due to photosphere-light abnormally refracted in the atmosphere of metallic vapours surrounding the sun: in other words, the light of the flashspectrum does not come from the reversing layer at all, but from the photosphere. The author shows that the light which will be thus abnormally refracted will be of wave-lengths almost identical with the wave-lengths which the metallic vapours are themselves capable of radiating. This beautiful theory not only explains the apparent shallowness of the reversing layer, a thing that has always puzzled astrophysicists, but it accounts for the extraordinary brilliancy of the lines.

I have succeeded in producing such a flash-spectrum by an arrangement in which I have endeavoured to imitate as closely as possible the conditions supposed to exist at the surface of the sun : in brief, I have obtained a spectrum of bright lines, with light from a source showing a continuous spectrum, by means of anomalous dispersion in an incandescent metallic vapour.

The theory of Julius supposes the sun to be surrounded by an atmosphere of metallic vapours, the refractive index of which decreases with increasing distance from the surface. In this atmosphere the rays of light coming from the photosphere will move in curved paths similar to rays in our own atmosphere.

The refractive index is, however, very small except for

* Communicated by the Physical Society : read April 26, 1901. 